\title{
Pairwise Error Probability Analysis of Low Density Parity Check Coded Asynchronous Distributed Space Time Block Codes
}

\author{
Varsha Vimal Sood, Surbhi Sharma, Rajesh Khanna \\ Department of Electronics and Communication Engineering, Thapar Institute of Engineering and \\ Technology, \\ Patiala, Punjab 147004, India \\ varsha.vimal@thapar.edu
}

\begin{abstract}
In this work, we have analysed the performance of a low density parity check (LDPC) coded Amplify and forward (AF) based asynchronous distributed space time block coded (ADSTBC) cooperative system. The proposed concatenated LDPC-ADSTBC is delay tolerant, where LDPC codes provide coding gain to the system and ADSTBC provides diversity gain for arbitrary number of relays, number of symbols transmitted and number of receive antennas. For the multi-relay scenario, we have derived closed form expression for the pair-wise error probability (PEP) of the system. The derived analytical results of the performance of the proposed delay tolerant multiple relay asynchronous cooperative communication are validated by simulation results.
\end{abstract}

Index Terms-Asynchronous communication; Channel coding; Cooperative communication; Pair wise error probability.

\section{INTRODUCTION}

Future wireless networks rely heavily on multiple input multiple output (MIMO) as one of its strongest potential candidate [1]. MIMO systems are an efficient technique offering diversity and multiplexing gains in comparison with single antenna systems without increasing demands for radio resources [2]. In many wireless scenarios there is a limitation on power, size, and hardware complexity and propagation environment which limit the deployment of multiple antennas as in MIMO. Cooperative communication [3-5] is a technique to overcome these drawbacks and form a virtual MIMO antenna array by utilizing relays which assist the direct communication with high diversity gain. Linear dispersion (LD) codes [6] act as an umbrella codes yielding both diversity and capacity gains for synchronous as well as the asynchronous environments. These were later extended to the cooperative communications as Distributed space Time Block codes (DSTBC) [7]. Due to its distributed topology, the DSTBC systems are basically asynchronous in nature [8]. For the codes to yield diversity gains, they have to be delay tolerant, i.e. full diversity should be satisfied for any delay order. Several delay tolerant techniques have been proposed in existing literature [9]-[12]. The delay tolerant schemes although provide diversity gains, are deficient in

Manuscript received 8 May, 2018; accepted 23 September, 2018 coding gain which can be achieved using channel codes such as Low density parity check (LDPC) codes. LDPC codes are highly efficient linear error-correcting codes.

Existing literature consists of several techniques in cooperative communication concatenated with LDPC codes thereby yielding enhanced performance of the system in the synchronous environment [13]-[16]. But the literature lacks the study and analysis of the asynchronous cooperative system with LDPC codes. The given research gap has been exploited as a motivation for the presented work.

In this paper, we have derived the closed form expression for the pair wise error probability (PEP) of asynchronous DSTBC (ADSTBC) system concatenated with an irregular LDPC codes. The novelty of the present work lies in the fact that the proposed ADSTBC system with ' $R$ ' relays can achieve significant diversity gain as well as coding gain by applying LDPC at transmitter. The LD codes used at the relays linearly modulate the received signal and retransmit ' $\mathrm{N}$ ' symbols in ' $\mathrm{T}$ ' channel uses. For $\mathrm{N}>\mathrm{T}$, the proposed system achieves a rate higher than unity. Also, the closed form expression for the pair wise error probability (PEP) of delay tolerant ADSTBC system concatenated with an irregular LDPC codes has been derived. The remaining of this paper is organized as follows. In Section II, the system model of the proposed low density parity checkasynchronous distributed space time block codes (LDPCADSTBC) system is described. The performance analysis of the proposed system with respect to the PEP of the system is done in Section III. The results are discussed in detail in Section IV. Section V concludes the paper.

\section{SYSTEM MODEL}

In this work an LDPC-ADSTBC system has been proposed constituting of ' $\mathrm{R}+2$ ' nodes, where the transmitter and each of the $\mathrm{R}$ relay nodes have single antenna whereas the receiver can employ variable number of antennas. A ( $J, K)$ LDPC code having code rate $1 / 2$ is employed here, where $\mathrm{K}$ refers to the length of the information vector and $\mathrm{J}$ is the length of the LDPC codeword. The cooperative communication process can be analysed in two phases. In the first phase, information bits are transmitted to the channel coder. A binary LDPC 
codeword corresponds to $\beta=\left(b_{1}, b_{2} \ldots . . b_{J}\right)$. The codeword is further BPSK modulated as $\mathbf{s}=\left[s_{1} \ldots \ldots s_{J}\right]$ in the codebook $\left\{\mathbf{s}_{1} \ldots . . . \mathbf{s}_{L}\right\}$ where $s_{J}=2 b_{J}-1$. The average power used at the transmitter for any transmission is assumed to be $P_{1}$. The transmitter broadcasts $\mathrm{N}$ symbols out of $\mathrm{J}$ symbols to the $\mathrm{R}$ relays in every transmission period. The signal received at $i^{\text {th }}$ relay is subjected to Rayleigh fading as well as the additive white Gaussian noise. Both the fading coefficients $f_{i}$ and the noise $\mathbf{n}_{i}$ are circularly symmetric Gaussian distributed with mean 0 and variance 1 . The signals received at the $i^{t h}$ relay is given as

$$
\mathbf{r}_{i}=\sqrt{P T} f_{i} \mathbf{s}+\mathbf{n}_{i}
$$

In the second phase, $\mathbf{x}_{i}=\left[x_{i, 1 \ldots .} x_{i, N}\right]$ are are transmitted by the $i^{\text {th }}$ relay to the destination. The receiver is assumed to have full knowledge of channel. The transmitted signal $\mathbf{x}_{i}$ from the $i^{\text {th }}$ relay is a combination of a certain LD matrix $\mathbf{A}_{i}$ and the incoming signal $\mathbf{r}_{i}$

$$
\mathbf{x}_{i}=\sqrt{\frac{P_{2}}{P_{1}+1}} \mathbf{A}_{i} \mathbf{r}_{i}
$$

where $P_{2}$ is the average transmitting power of relay. Each LD matrix $\mathbf{A}_{i}$ should conform to $\mathbf{A}_{i} \mathbf{A}_{i}^{H}=\mathbf{I}$ (where $\mathbf{I}$ is an identity matrix of size $\mathrm{T} \times \mathrm{T}$ ). $\mathrm{T}$ is the coherence time of phase I, when the channel fading coefficients remain constant. The dispersion matrix $\mathbf{A}_{i}$ distributes the $\mathrm{N}$ symbols among $\mathrm{T}$ channel uses. The received signal $\mathbf{y}(t)$ at the $n^{\text {th }}$ antenna of the receiver at time $\mathrm{t}$ is given by

$$
\mathbf{y}(t)=\sum_{i=1}^{R} \sum_{n=1}^{N_{d}} g_{i, n}(t) \mathbf{x}\left(t-\delta_{i}\right)+\mathbf{v}_{n}(t)
$$

where $\mathbf{G}=\left[\begin{array}{ccc}g_{11} & \cdots & g_{1, N_{d}} \\ \vdots & \ddots & \vdots \\ g_{R, N_{d}} & \cdots & g_{R, N_{d}}\end{array}\right]$

The Rayleigh fading channel coefficient $g_{i, n}$ is the $(i, n)^{t h}$ entry of the matrix $\mathbf{G}$ for $i \leq R ; 1 \leq n, N_{d}$ is the number of receive antennas at the destination $\mathbf{v}_{n}(t)$ is the additive white Gaussian noise with variance $N_{0} . \delta_{i}$ is the delay observed for the $i^{\text {th }}$ relay at the receiver relative to the first relay. The delay profile can be estimated from the arrival of the pilot signals. The receiver is synchronized to the first relay i.e. $\delta_{1}=0$. The relative delay profile of the ADSTBC system is denoted as $\Delta=\left(\delta_{1} \ldots \ldots \delta_{R}\right)$ and $\delta_{\max }=\max \left\{\delta_{1} \ldots . \delta_{R}\right\}$. For the code to be delay tolerant, (3) is written as

$$
\mathbf{y}=\sum_{i=1}^{R} \sum_{n=1}^{N_{d}} g_{i, n} \mathbf{D}\left(\delta_{i}\right) \mathbf{t}_{i}+\mathbf{v}
$$

where and $\mathbf{D}\left(\delta_{i}\right)=\left\lfloor\begin{array}{c}\mathbf{O}_{\delta_{i} \times T} \\ \mathbf{I}_{T} \\ \mathbf{O}_{\left(\delta_{\max }-\delta_{i}\right) \times T}\end{array}\right\rfloor$ and $\mathbf{O}$ denote an all zero matrix. Putting (1) and (2) into (4) and solving further

$$
\begin{gathered}
\mathbf{y}=\sum_{i=1}^{R} \sum_{n=1}^{N_{d}} g_{i . n} \sqrt{\frac{P_{2}}{P_{1}+1}} \mathbf{D}\left(\delta_{i}\right) \mathbf{A}_{i}\left(\sqrt{P_{1} T} f_{i} \mathbf{s}+\mathbf{n}_{i}\right)+\mathbf{v}= \\
=\sqrt{\frac{P_{1} P_{2} T}{P_{1}+1}}(\hat{\mathbf{H}} \mathbf{D}(\Delta) \mathbf{C})+\mathbf{V}
\end{gathered}
$$

where $\quad \hat{\mathbf{H}}=\mathbf{H} \otimes \mathbf{I}, \quad \mathbf{H}=\left[\begin{array}{ccc}f_{1} g_{11} & \cdots & f_{1} g_{R 1} \\ \vdots & \ddots & \vdots \\ f_{1} g_{1 N_{d}} & \cdots & f_{1} g_{R N_{d}}\end{array}\right]$,

$\mathbf{D}(\Delta)=\operatorname{diag}\left(\mathbf{D}\left(\delta_{1}\right), . . \mathbf{D}\left(\delta_{R}\right)\right), \quad \mathbf{C}=\left[\left(\mathbf{A}_{1} \mathbf{s}\right)^{\prime} \cdots\left(\mathbf{A}_{R} \mathbf{s}\right)^{\prime}\right]^{\prime} . \quad \mathbf{H}$ represents the overall channel gain from the source to the destination. $\mathbf{C}$ is the LD codeword formed by the cooperation of all the $\mathrm{R}$ relays. $\left(\mathbf{A}_{i} \mathbf{s}\right)^{\prime}$ denotes the transform of $\left(\mathbf{A}_{i} \mathbf{s}\right)$, and $\mathbf{V}=\sqrt{\frac{P_{2}}{P_{1}+1}} \sum_{i=1}^{R} \sum_{n=1}^{N_{d}} g_{i, n} \mathbf{D}\left(\delta_{i}\right) \mathbf{A}_{i} \mathbf{n}_{i}+\mathbf{v}$. The matrix $\mathbf{C}$ having dimensions $\mathrm{R} \times \mathrm{T}$ corresponds to the space time code as in a MIMO system but since being generated in a distributive manner is called a distributed space time code (DSTBC). Manipulating (5) for the vertical stacking of the columns of $\mathbf{y}$ gives the following equivalent matrix

$$
\overline{\mathbf{y}}=\sqrt{\frac{P_{1} P_{2} T}{P_{1}+1}} \overline{\mathbf{H}} \mathbf{D}(\Delta) \overline{\mathbf{\chi}} \overline{\mathbf{s}}+\overline{\mathbf{V}}=\sqrt{\frac{P_{1} P_{2} T}{P_{1}+1}} \overline{\mathbf{H}} \overline{\mathbf{C}}+\overline{\mathbf{V}},
$$

where $\overline{\mathbf{H}}=\mathbf{H} \otimes \mathbf{I}_{\Delta}, \quad \mathbf{I}_{\Delta}=$ identity matrix of size $(\mathrm{T}+$ maximum delay), $\overline{\mathbf{C}}=\mathbf{D}(\Delta) \overline{\boldsymbol{\chi}} \overline{\mathbf{s}}$ represent the delay tolerant ADSTBC codeword. $\bar{\chi}=\operatorname{diag}\left(\mathbf{A}_{1}, \ldots, \mathbf{A}_{R}\right) \quad$ and $\overline{\mathbf{s}}=\left[\begin{array}{lll}\mathbf{s} & \cdots & \mathbf{s}\end{array}\right]^{\prime}$, where $\mathbf{s}$ is stacked $\mathrm{R}$ number of times. The transmitted symbols are detected using a maximum likelihood (ML) decoder as

$$
\hat{\overline{\mathbf{s}}}=\arg \min _{s_{k}}\left\|\overline{\mathbf{y}}-\sqrt{\frac{P_{1} P_{2} T}{P_{1}+1} \overline{\mathbf{H}}} \overline{\mathbf{C}}\right\|^{2} .
$$

The output of the ML decoder is given to the LDPC decoder which outputs the estimated information vector. The output can be analysed for various parameters. In this paper we focus on the error performance, particularly the pair wise error probability (PEP).

\section{PERFORMANCE ANALYSIS OF LDPC-ADSTBC SYSTEM}

\section{A. Pair Wise Error Probability}

We begin by deriving the PEP for LDPC-ADSTBC with two relays (i.e. $\mathrm{R}=2$ ). Let $\mathbf{S}=\left\{\overline{\mathbf{C}}_{k}\right\}$ denote the LDPCADSTBC codeword set, where $1 \leq k \leq|\mathbf{S}|$ and $|\mathbf{S}|$ denotes the cardinality of the codeword set $\mathbf{S}$. Let 
$P\left(\overline{\mathbf{C}}_{k} \rightarrow \overline{\mathbf{C}}_{l} / \mathbf{H}, \boldsymbol{\Delta}\right)$ be the probability of mistaking $\overline{\mathbf{C}}_{k}$ by $\overline{\mathbf{C}}_{l}$, where $\overline{\mathbf{C}}_{k}$ and $\overline{\mathbf{C}}_{l}$ are elements in the ADSTBC set conditioned on the fading channel matrix $\mathbf{H}$ and the delay profile $\Delta$ is given as

$$
P\left(\frac{\overline{\mathbf{C}}_{k} \rightarrow \overline{\mathbf{C}}_{l}}{\mathbf{H}, \boldsymbol{\Delta}}\right)=Q\left(\sqrt{\frac{P_{1} P_{2} T}{\left(P_{1}+1\right)} \frac{\left\|\overline{\mathbf{H}}\left(\overline{\mathbf{C}}_{k}-\overline{\mathbf{C}}_{l}\right)\right\|_{F}^{2}}{2\left(\frac{P_{2}}{P_{1}+1} \sum_{i=1}^{R}\left|g_{i, n}\right|^{2}+1\right)}}\right)
$$

The Frobenius norm $\left\|\overline{\mathbf{H}}\left(\overline{\mathbf{C}}_{k}-\overline{\mathbf{C}}_{l}\right)\right\|_{F}^{2}$ can also be written as given by [2]

$$
\begin{aligned}
\operatorname{Tr}\left\{\overline{\mathbf{H}}\left(\overline{\mathbf{C}}_{k}-\overline{\mathbf{C}}_{l}\right)\left(\overline{\mathbf{C}}_{k}-\overline{\mathbf{C}}_{l}\right)^{H} \overline{\mathbf{H}}^{H}\right\}=\operatorname{Tr}\left\{N_{k l} \tilde{\mathbf{H}} \tilde{\mathbf{H}}^{H}\right\}= \\
=\sum_{i=1}^{R} \lambda_{k, i} \sum_{\tilde{i}=1}^{R}\left|\tilde{h}_{\tilde{i}, i}\right|^{2},
\end{aligned}
$$

where $\left(\left(\overline{\mathbf{C}}_{k}-\overline{\mathbf{C}}_{l}\right)\left(\overline{\mathbf{C}}_{k}-\overline{\mathbf{C}}_{l}\right)^{H}\right)=M_{k l}$ is the code word difference matrix. $(\mathbf{D}(\Delta) \bar{\chi})^{H}(\mathbf{D}(\Delta) \bar{\chi})=\mathbf{I}$, coefficient $\tilde{h}_{\tilde{i}, i}$ is the $(\tilde{i}, i)^{t h}$ entry of the matrix $\tilde{\mathbf{H}}=\mathbf{H D}(\Delta) \bar{\chi}$ for $N_{k l}=\left[\begin{array}{ccc}\lambda_{k, 1} & 0 & 0 \\ 0 & \ddots & 0 \\ 0 & 0 & \lambda_{k, R}\end{array}\right]$ contains the Eigen values, $\lambda_{k, 1}, \lambda_{k, 2}$ of the codeword difference matrix $M_{k l}$. By substituting (9) for $\left\|\overline{\mathbf{H}}\left(\overline{\mathbf{C}}_{k}-\overline{\mathbf{C}}_{l}\right)\right\|_{F}^{2}$ in (8) we obtain

$$
P\left(\frac{\overline{\mathbf{C}}_{k} \rightarrow \overline{\mathbf{C}}_{l}}{\mathbf{H}, \boldsymbol{\Delta}}\right)=Q\left(\sqrt{\frac{P_{1} P_{2} T \sum_{i=1}^{R} \lambda_{k, i} \sum_{i=1}^{R}\left|\tilde{h}_{\tilde{i}, i}\right|^{2}}{2\left(\frac{P_{2}}{\left.P_{1}+1\right)} \sum_{i=1}^{R}\left|g_{i, n}\right|^{2}+1\right)}}\right)
$$

The unconditional PEP over $\mathbf{H}$ is given by

$$
\left.P\left(\frac{\overline{\mathbf{C}}_{k} \rightarrow \overline{\mathbf{C}}_{l}}{\Delta}\right)=Q \sqrt{\frac{P_{1} P_{2} T \sum_{i=1}^{R} \lambda_{k, i} \sum_{i=1}^{R}\left|\tilde{h}_{\tilde{i}, i}\right|^{2}}{2\left(\frac{\left.P_{2}+1\right)}{P_{1}+1} R+1\right)}}\right),
$$

where $\tilde{h}_{\tilde{i}, i}$ are the entries of the Rayleigh distributed effective channel matrix $\tilde{H}$. Re-writing the above equation using Craig's bound where:

$$
\begin{gathered}
Q(x)=\frac{1}{\pi} \int_{0}^{\frac{\pi}{2}} e^{-\frac{x^{2}}{2 \sin ^{2} \theta}} d \theta, \\
P\left(\frac{\overline{\mathbf{C}}_{k} \rightarrow \overline{\mathbf{C}}_{l}}{\Delta}\right) \leq \frac{1}{2} \int_{0}^{\frac{\pi}{2}} e^{-\frac{P_{1} P_{2} T G \sum_{i=1}^{R} \lambda_{k, i}}{4\left(1+P_{1}+R P_{2}\right) \sin ^{2} \theta}} d \theta,
\end{gathered}
$$

where $G$ denotes the average power gain of the coefficients. Further the unconditional average PEP w.r.t. all delay profiles is given as

$$
\begin{gathered}
E_{\Delta}\left\lfloor P\left(\frac{\overline{\mathbf{C}}_{k} \rightarrow \overline{\mathbf{C}}_{l}}{\Delta}\right)\right\rfloor= \\
=\psi\left(\prod_{i=1}^{R}\left(1+\frac{P_{1} P_{2} T G \lambda_{k, i}}{4\left(1+P_{1}+R P_{2}\right) \sin ^{2} \theta}\right)\right),
\end{gathered}
$$

where $\psi(\eta(\theta))=\frac{1}{\pi} \int_{0}^{\frac{\pi}{2}} \frac{1}{\eta(\theta)} d \theta$. The total PEP for decoding at the destination can be upper bounded using the union bound which is the sum of the PEPs over all code words $\overline{\mathbf{C}}_{k} \in \mathbf{S}$ is given as

$$
P_{k l} \leq \sum_{k=1}^{|\mathbf{S}|} \psi\left(\prod_{i=1}^{R}\left(1+\frac{P_{1} P_{2} T G \lambda_{k, i}}{4\left(1+P_{1}+R P_{2}\right) \sin ^{2} \theta}\right)\right)
$$

For high SNR the total PEP can be approximated as

$$
P_{k l} \leq \sum_{k=1}^{|S|} \psi\left(\frac{P_{1} P_{2} T G}{4\left(1+P_{1}+R P_{2}\right)} \prod_{i=1}^{R}\left(\lambda_{k, i}\right)^{r}\right)
$$

where $\mathrm{r}$ is the rank of codeword difference matrix $M_{k l}$ and equals $\mathrm{R}$ for all delay profiles in case of full diversity ADSTBC [9]. The result can be extended to multiple antennas receiver with number of antennas equal to $N_{d}$ and achieving full diversity is given as follows

$$
P_{k l} \leq \sum_{k=1}^{|S|} \psi\left(\left(\frac{P_{1} P_{2} T G}{4\left(1+P_{1}+R P_{2}\right)}\right)^{N_{d}} \prod_{i=1}^{r} \lambda_{k, i}^{r N_{d}}\right) .
$$

\section{RESUlTS AND DisCUSSIONS}

The analytical results have been obtained for the LDPCADSTBC system for single transmitter-receiver pair and $\mathrm{R}$ relays. We assume that the source and relays are equipped with single antenna each whereas the receiver has variable number of antennas, $N_{d}$. In this paper an irregular LDPC with column weight 3 has been employed. Monte- Carlo simulations using MATLAB validate the analytical results obtained using Mathematica. A frame consisting of LDPC coded bits is sent from the LDPC encoder with all the bits with random values 0 and 1 which has further been BPSK modulated and broadcast to the relays. The results have been obtained from 1000 independent iterations of each frame, 
for each value of SNR varying from $0 \mathrm{~dB}$ to $20 \mathrm{~dB}$. Figure 1 shows the plot of error performance of the synchronous DSTBC, ADSTBC and LDPC-ADSTBC with varying $N_{d}$ at the receiver. It can be observed that, at PEP of $10^{-3}$ for single antenna, the synchronous DSTBC requires an SNR of $19.25 \mathrm{~dB}$, the ADSTBC requires $16.25 \mathrm{~dB}$ whereas LDPC coded the ADSTBC requires $14.75 \mathrm{~dB}$. The comparison among the transmission techniques has been tabulated in Table I. We observe an improvement of $1.5 \mathrm{~dB}$ in SNR for PEP of $10^{-3}$ for the case of LDPC coded asynchronous DSTBC system (LDPC-ADSTBC) over asynchronous DSTBC system and $4.5 \mathrm{~dB}$ over synchronous DSTBC. When, $N_{d}$ is increased from a single antenna to 2 , we observe an increase in diversity as well as coding gain. At same PEP for two antennas, SNR required by synchronous DSTBC, ADSTBC and LDPC-ADSTBC shows an improvement of $1.25 \mathrm{~dB}$ in SNR for the case LDPCADSTBC over ADSTBC system and $4.5 \mathrm{~dB}$ over synchronous DSTBC.

TABLE I. SNR REQUIRED FOR DIFFERENT TRANSMISSION SCHEMES FOR $\mathrm{N}=\mathrm{R}=2$ AND VARING $N_{D}$ AT PEP OF $10^{-3}$

\begin{tabular}{|c|c|c|}
\hline \multirow{2}{*}{ Scheme } & \multicolumn{2}{|c|}{ SNR required in $\mathrm{dB}$ at PEP of $10^{-3}$} \\
\cline { 2 - 3 } & $N_{d}=1$ & $N_{d}=2$ \\
\hline DSTBC & 19.25 & 13 \\
\hline ADSTBC & 16.25 & 9 \\
\hline LDPC-ADSTBC & 14.75 & 8.25 \\
\hline
\end{tabular}

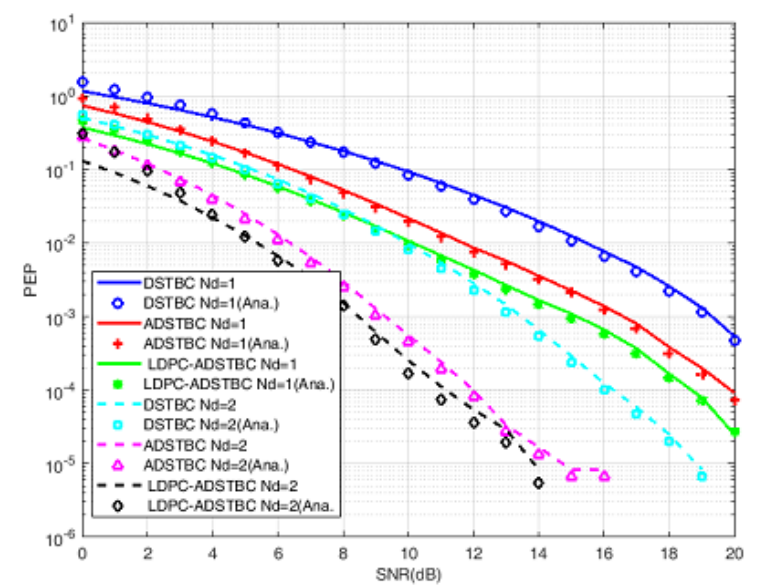

Fig. 1. Error performance of different systems by varying number of antennas $\left(N_{d}\right)$ at the receiver.

Figure 2 shows the PEP of the LDPC-ADSTBC with delay of two and one symbol period at the two relay nodes with number of modulated symbols $=2$ and 3 and $N_{d}=1$ and 2. From Table II, it is observed that, for the given PEP, LDPC-ADSTBC with $\mathrm{N}=2, N_{d}=1$ requires an $\mathrm{SNR}$ of $14.75 \mathrm{~dB}$ whereas the LDPC-ADSTBC $\mathrm{N}=2, N_{d}=2$ requires an SNR of $8.25 \mathrm{~dB}$. When we increase the number of modulated symbols from 2 to 3 the transmission rate increases thereby leading to a fall in diversity. However the SNR required for $\mathrm{N}=3$, in case of $\mathrm{LDPC}-\mathrm{ADSTBC} \mathrm{N}=3$, $N_{d}=1$ is approximately $17 \mathrm{~dB}$ whereas in the LDPC$\operatorname{ADSTBC~} \mathrm{N}=3, N_{d}=2$ case an SNR of $9.5 \mathrm{~dB}$ is required.

TABLE II. SNR REQUIRED FOR LDPC-ADSTBC FOR VARYING N AND $N_{D}$ AND FIXED R AT PEP OF $10^{-3}$.

\begin{tabular}{|c|c|c|}
\hline No. of modulated & \multicolumn{2}{|c|}{ SNR required in $\mathrm{dB}$ at PEP of $10^{-3}$} \\
\cline { 2 - 3 } symbols (N) with $\mathbf{R}=\mathbf{2}$ & $N_{d}=1$ & $N_{d}=2$ \\
\hline $\mathbf{N}=\mathbf{2}$ & 14.75 & 8.25 \\
\hline $\mathbf{N}=\mathbf{3}$ & 17 & 9.5 \\
\hline
\end{tabular}

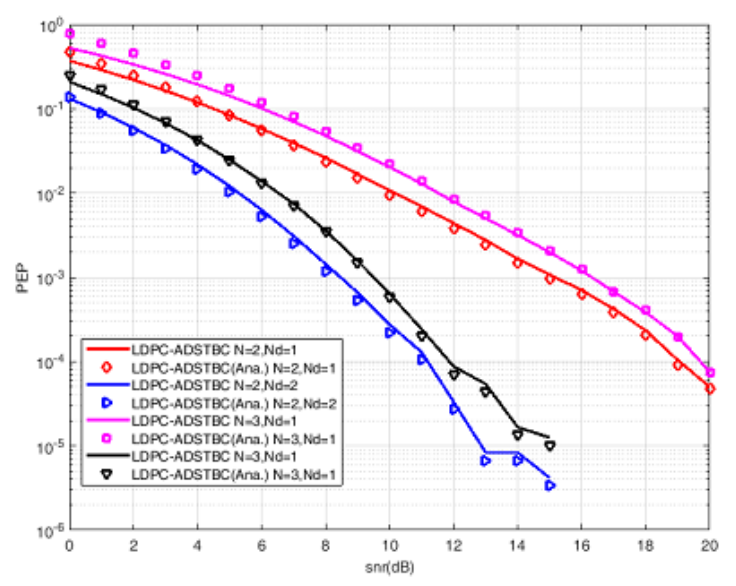

Fig. 2. Error performance of the LDPC-ADSTBC with delay $(0,1)$, no. of symbols $=2$ and 3 and no. of antennas at the receiver $\left(N_{d}\right)=1$ and 2 respectively.

From Fig. 3 it can be observed that there is significant improvement in error performance of the LDPC-ADSTBC with 3 relays $(\mathrm{R})$ and 2 antennas at the receiver and the asynchronous LDPC-DSTBC with 2 relays and 1 antenna at the receiver.

In Table III, it is evident that, for the given PEP and $N_{d}=$ 1 , the SNR required by LDPC-ADSTBC at $\mathrm{R}=2$ and 3 are $14.75 \mathrm{~dB}$ and $11.5 \mathrm{~dB}$ respectively, whereas with $N_{d}=2$, the required SNR decreases by 6.25 and 4.5 respectively. The performance of the LDPC-ADSTBC system approximates the results of [2], which is the case of PEP of synchronous scenario, even in the asynchronous environment.

TABLE III. SNR REQUIRED FOR LDPC-ADSTBC FOR VARYING R AND $N_{D}$ AND FIXED N AT PEP OF $10^{-3}$

\begin{tabular}{|c|c|c|}
\hline No. of relays $(\mathbf{R})$ with & \multicolumn{2}{|c|}{ SNR required in $\mathrm{dB}$ at PEP of $10^{-3}$} \\
\cline { 2 - 3 } $\mathbf{N}=\mathbf{2}$ & $N_{d}=1$ & $N_{d}=2$ \\
\hline $\mathbf{R}=\mathbf{2}$ & 14.75 & 8.5 \\
\hline $\mathbf{R}=\mathbf{3}$ & 11.5 & 7 \\
\hline
\end{tabular}

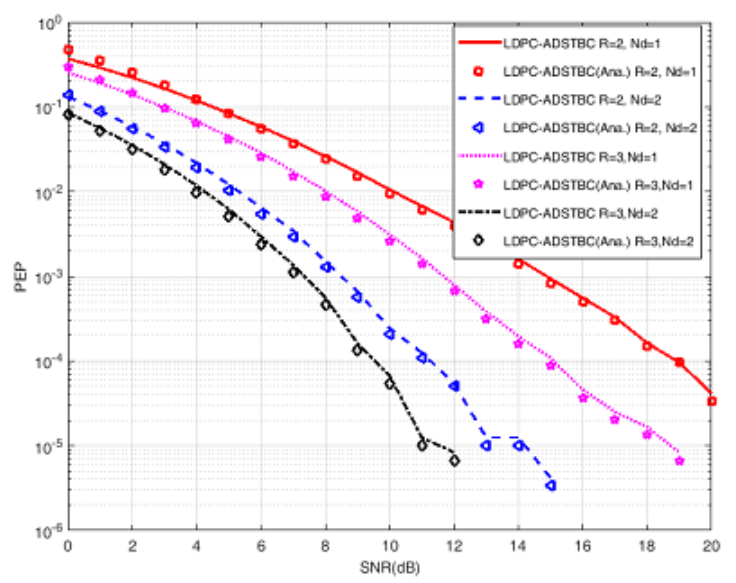

Fig. 3. Error performance of the LDPC-ADSTBC with $\mathrm{R}=2,3$ and $N_{d}=1$, 2 .

\section{CONCLUSIONS}

In this paper, we have analysed the error performance of LDPC codes concatenated with ADSTBC system. The PEP of the system under study is analysed using Craig's bounds. Analytical results verify the performance of the LDPCADSTBC system. We obtain an SNR gain of over $5 \mathrm{~dB}$ with LDPC-ADSTBC as compared to synchronous DSTBC system. Performance further improves by increasing number of relays. It is concluded that LDPC-ADSTBC codes perform better than their synchronous counterpart. Design 
and performance analysis of the present work in different fading environments has been proposed as future work.

\section{REFERENCES}

[1] G. Naik, J. Liu, J. M. Park, "Coexistence of wireless technologies in the $5 \mathrm{GHz}$ bands: a survey of existing solutions and a roadmap for future research", IEEE Comm. Surveys \& Tutorials, vol. 20, no. 3, pp. 1777-1798, 2018. DOI: 10.1109/COMST.2018.2815585.

[2] N. Varshney, A. V. Krishna, A. K. Jagannatham, "Selective DF protocol for MIMO STBC based single/multiple relay cooperative communication: end-to-end performance and optimal power allocation", IEEE Trans. Comm., vol. 63, no. 7, pp. 2458-2474, 2015. DOI: 10.1109/TCOMM.2015.2436912.

[3] Q. Li, R. Q. Hu, Y. Qian, G. Wu, "Cooperative communications for wireless networks: techniques and applications in LTE-advanced systems", IEEE Wireless Comm, vol. 19, no. 2, 2012. DOI: 10.1109/MWC.2012.6189409.

[4] V. Markevičius, D. Navikas, D. Andriukaitis, M. Čepėnas, A. Valinevičius, M. Žilys, R. Malekian, A. Janeliauskas, W. Walendziuk, A. Idzkowski, "Two thermocouples low power wireless sensors network", AEU-International journal of electronics and communications, vol. $84, \quad$ pp. $242-250, \quad 2017 . \quad$ DOI: 10.1016/j.aeue.2017.11.032.

[5] H.-S. Nguyen, M.-T. Nguyen, M. Voznak, "Successful transmission probability of cognitive device-to-device communications underlying cellular networks in the presence of hardware impairments", Eurasip Journal on Wireless Communications and Networking, vol. 208, 2017. DOI: 10.1186/s13638-017-0994-0.

[6] N. Wu, H. Gharavi, "Asynchronous cooperative MIMO systems using a linear dispersion structure", IEEE Trans. Veh Tech., vol. 59, no. 2 , pp. 779-787, 2010. DOI: 10.1109/TVT.2009.2036434.

[7] T. Peng, R. C. de Lamare, "Adaptive buffer-aided distributed space- time coding for cooperative wireless networks", IEEE Trans. Comm., vol. 64, no. 5, pp. 1888-1900, 2016. DOI: 10.1109/TCOMM.2016.2544934.

[8] Shuangqing Wei, D. L. Goeckel, M. C. Valenti, "Asynchronous cooperative diversity", IEEE Trans. Wireless Comm., vol. 5, no. 6, pp. 1547-1557, 2006. DOI: 10.1109/TWC.2006.1638675.

[9] W. Wang, F. C. Zheng, M. Fitch, "Design of delay-tolerant spacetime codes with limited feedback", IEEE Trans. Veh. Tech., vol. 64 no. 2, pp. 839-845, 2015. DOI: 10.1109/TVT.2014.2322623.

[10] R. Cao, F. Qu, L. Yang, "Asynchronous amplify-and-forward relay communications for underwater acoustic networks", IET Comm. vol. 10, no. 6, pp. 677-684, 2016. DOI: 10.1049/iet-com.2014.1233.

[11] J. Wang, Q. Yu, Z. Li, C. Bi, "Distributed space time block transmission and QRD based diversity detector in asynchronous cooperative communications systems", IEEE Trans. Vehicular Technology, vol. 67, no. 6, pp. 5111-5125, 2018. DOI 10.1109/TVT.2018.2812901

[12] B. S. Kim, K. Choi, "FADAC-OFDM: frequency-asynchronous distributed alamouti-coded OFDM", IEEE Trans. Veh. Tech., vol. 64, no. 2, pp. 466-480, 2015. DOI: 10.1109/TVT.2014.2325606.

[13] M. Rahmati, T. M. Duman, "Spectrally efficient alamouti code structure in asynchronous cooperative systems", IEEE Signal Processing Letters, vol. 21, no. 5, pp. 545-549, 2014. DOI: 10.1109/LSP.2014.2308296.

[14] S. Mehrizi, S. Khosravi, M. Ahmadian, "An efficient procedure for bilayer-expurgated LDPC codes design in cooperative relay channels", IEEE Comm. Letters, vol. 21, no. 10, pp. 2114-2117, 2017. DOI: 10.1109/LCOMM.2017.2708699.

[15] H. Khodaiemehr, D. Kiani, M. R. Sadeghi, "LDPC lattice codes for full-duplex relay channels", IEEE Trans. Comm., vol. 65, no. 2, pp. 536-548, 2017. DOI: 10.1109/TCOMM.2016.2638839.

[16] D. N. K. Jayakody, "LDPC coded lossy forwarding scheme for cooperative wireless networks", Electronics Letters, vol. 52, no. 25 , pp. 2070-2072, 2016. DOI: 10.1049/el.2016.2444. 